\title{
A biochemical/biophysical 3D FE intervertebral disc model
}

\author{
Y. Schroeder · J. M. Huyghe - C. C. van Donkelaar • \\ K. Ito
}

Received: 8 October 2009 / Accepted: 23 February 2010 / Published online: 13 March 2010

(C) The Author(s) 2010. This article is published with open access at Springerlink.com

\begin{abstract}
Present research focuses on different strategies to preserve the degenerated disc. To assure long-term success of novel approaches, favorable mechanical conditions in the disc tissue are essential. To evaluate these, a model is required that can determine internal mechanical conditions which cannot be directly measured as a function of assessable biophysical characteristics. Therefore, the objective is to evaluate if constitutive and material laws acquired on isolated samples of nucleus and annulus tissue can be used directly in a wholeorgan 3D FE model to describe intervertebral disc behavior. The 3D osmo-poro-visco-hyper-elastic disc (OVED) model describes disc behavior as a function of annulus and nucleus tissue biochemical composition, organization and specific constituent properties. The description of the 3D collagen network was enhanced to account for smaller fibril structures. Tissue mechanical behavior tests on isolated nucleus and annulus samples were simulated with models incorporating tissue composition to calculate the constituent parameter values. The obtained constitutive laws were incorporated into the whole-organ model. The overall behavior and disc properties of the model were corroborated against in vitro creep experiments of human L4/L5 discs. The OVED model simulated isolated tissue experiments on confined compression and uniaxial tensile test and whole-organ disc behavior. This was possible, provided that secondary fiber structures were accounted for. The fair agreement (radial bulge, axial creep deformation and intradiscal pressure) between model and experiment was obtained using constitutive properties that are the same for annulus and nucleus. Both tissue models differed in the 3D OVED model only by composition.
\end{abstract}

Y. Schroeder · J. M. Huyghe $(\varangle) \cdot$ C. C. van Donkelaar · K. Ito Department of Biomedical Engineering,

Eindhoven University of Technology, P.O. Box 513,

$5600 \mathrm{MB}$, Eindhoven, The Netherlands

e-mail: j.m.r.huyghe@tue.nl; jacques@wfw.wtb.tue.nl
The composition-based modeling presents the advantage of reducing the numbers of material parameters to a minimum and to use tissue composition directly as input. Hence, this approach provides the possibility to describe internal mechanical conditions of the disc as a function of assessable biophysical characteristics.

Keywords Intervertebral disc - Finite element analysis . Osmolarity $\cdot$ Poroviscoelasicity $\cdot$ Biochemistry

\section{Introduction}

Back pain is a frequently occurring complaint in adults, having a relatively large impact on the European economy as it often partially incapacitates the patient (Op De Beeck and Hermans 2000). Degeneration in the disc, which in some cases is associated with back pain, occurs for reasons that are still unclear. The diagnosis and treatment of painful degenerative disc disease remain one of the most controversial topics in spinal research, as current treatment aims at reducing pain rather than repairing the degenerated disc (Urban and Roberts 2003). Various approaches ranging from conservative to surgical treatments (e.g. total removal of disc, spinal fusion) are employed, which are less than adequate (Roughley 2004). Thus, present research focuses on different strategies to preserve the disc fully or partially, e.g., dynamic stabilization, biological remodeling or nucleus prosthesis. Favorable mechanical conditions in the disc tissue are essential to assure the long-term success of these novel approaches. To evaluate these conditions, a model is required that simulates internal mechanical conditions which cannot be directly measured as a function of biophysical characteristics which can be directly determined. 
Since measurements in living humans are complex, dangerous and impossible for some parameters, finite element (FE) models have become an important tool to study load distribution in healthy and degenerated discs (Adams et al. 1996; Brinckmann et al. 1983; Iatridis et al. 2003; Natarajan and Andersson 1999). Different finite element approaches have been made for the implementation of collagen fibers (Cheung et al. 2003; Wu and Chen 1996), and poroelastic models have been widely used to describe the time-dependent behavior of the disc tissue (Argoubi and Shirazi-Adl 1996; Natarajan and Andersson 1999). However, in current 3D disc models, changes associated with aging and degeneration have been described only phenomenologically, e.g., change in Young's modulus (Rohlmann et al. 2006; Ruberte et al. 2009; Schmidt et al. 2007). This is partly due to the limitation of most disc models where mechanical behavior of the tissue is not a result of our understanding of tissue mechanics. Iatridis et al. (2003) have shown that the influence of fixed charge density (FCD) on the mechanical, chemical and electrical behavior of the tissue can be taken into account with regard to modeling the disc. FCD is a measure of the swelling capacity of the tissue and underlines the role of osmotic forces in the disc behavior (Urban and Maroudas 1981). With increasing age and degeneration, biochemical composition of the structural components changes (Adams et al. 1996) and the load distribution between annulus and nucleus changes. This suggests that there is a direct correlation between material properties and biochemical composition of the tissue (Adams et al. 1996), which may be accounted for mechanistically in models.

The main structural components of the disc are nucleus pulposus and annulus fibrosus. The nucleus tissue behaves like a gel. Hydrophilic protoglycans (PG's) attract water through Donnan osmosis, but this swelling is restrained by the collagen network, resulting in a highly pressurized nucleus with high water content (Houben et al. 1997; Urban et al. 1979; Urban and Roberts 2003). On the other hand, the highly organized collagen structure of the annulus, i.e., 15-25 concentric lamellae that contain most of the fiber bundles arranged in alternating directions (Marchand and Ahmed 1990), resembles from a mechanical point of view a fiberreinforced composite material. One of the main advantages of this structure is the ability to withstand large and complex loads in multiple directions with optimized material proportions. The PG's intertwined in the collagen structure that attract water, contribute to the overall stiffness of the annulus as well as the smaller fibril structures present in the annulus, e.g., minor collagen, elastin or collagen cross-links (Cassidy et al. 1989; Guerin and Elliott 2006b; Matcher et al. 2004; Schollmeier et al. 2000). Recent experimental findings have demonstrated the complex interlamellar architecture of the annulus, with small collagen/elastin fibrils connecting the many fibers within the lamellae and connecting the lamellae to each other, as well as its relevance to disc biomechanics (Elliott and Setton 2000; Pezowicz et al. 2006b,a; Schollum et al. 2008; Veres et al. 2008). The mechanical properties and physical functions of the disc are mostly regulated by the combination of these two tissues (nucleus, annulus). In turn, the mechanical properties of each individual structural disc component are determined through their unique biochemical compositions and organization. However, the two tissues are composed of the same basic major biochemical components (water, collagen and PG's).

This understanding provided the ground base for the development of a disc FE model on the tissue level. The osmo-poro-visco-hyper-elastic disc (OVED) tissue model described the annulus and nucleus tissue as a function of its biochemical composition and organization. In an earlier study, the biochemical component water was closely evaluated, as experimental data indicated the importance of water differentiation (Sivan et al. 2006a; Urban and McMullin 1985). The total amount of water in the tissue is divided into intrafibrillar water (IFW) and extrafibrillar water (EFW). IFW is present in the intrafibrillar space within the collagen fibers and is, therefore, not accessible to the PG's, which reside in the extrafibrillar compartment. The IFW is especially of importance in the collagen-rich annulus fibrosus, because up to $30 \%$ of the total fluid may be IFW (Sivan et al. 2006b) which influences the swelling and load-bearing properties of the disc. Thus, the OVED tissue model was extended to include the intra and extrafibrillar water differentiation (Schroeder et al. 2007) and exhibited that the intradiscal pressure profile was clearly influenced by the IFW content (Schroeder et al. 2007). Quantification of IFW and EFW exchange is physiologically relevant, since gene expression of cells and propagation of cracks are affected by changes of extrafibrillar osmolarity (Chen et al. 2002; Neidlinger-Wilke et al. 2006; Wognum et al. 2006).

Constitutive laws acquired on isolated samples of nucleus and annulus tissue through confined compression experiments and uniaxial tensile tests enabled us to describe the anisotropic, non-linear behavior of the annulus and nucleus tissue (Schroeder et al. 2008). Accounting for the difference between nucleus and annulus tissue through its biochemical composition reduced the number of constituent parameters (Schroeder et al. 2008). This is of special interest, as variation in the experimental data entails a large variability in the material properties describing the disc tissue behavior (Jones and Wilcox 2008).

This paper presents a novel FE model at a wholeorgan level; the 3D OVED model describes the overall disc behavior as a function of annulus and nucleus tissue biochemical composition, organization and the specific material properties of each of these constituents (Schroeder et al. 2006; Schroeder et al. 2008). The description of the $3 \mathrm{D}$ collagen network in the $3 \mathrm{D}$ OVED model is 
Fig. 1 Finite element mesh of $1 / 4$ of an intervertebral disc with differentiation of nucleus (light) and annulus (dark) regions in the stress-free state, with selected nodes (white stars) on anterior, posterior and lateral side for later comparison (Table 1)

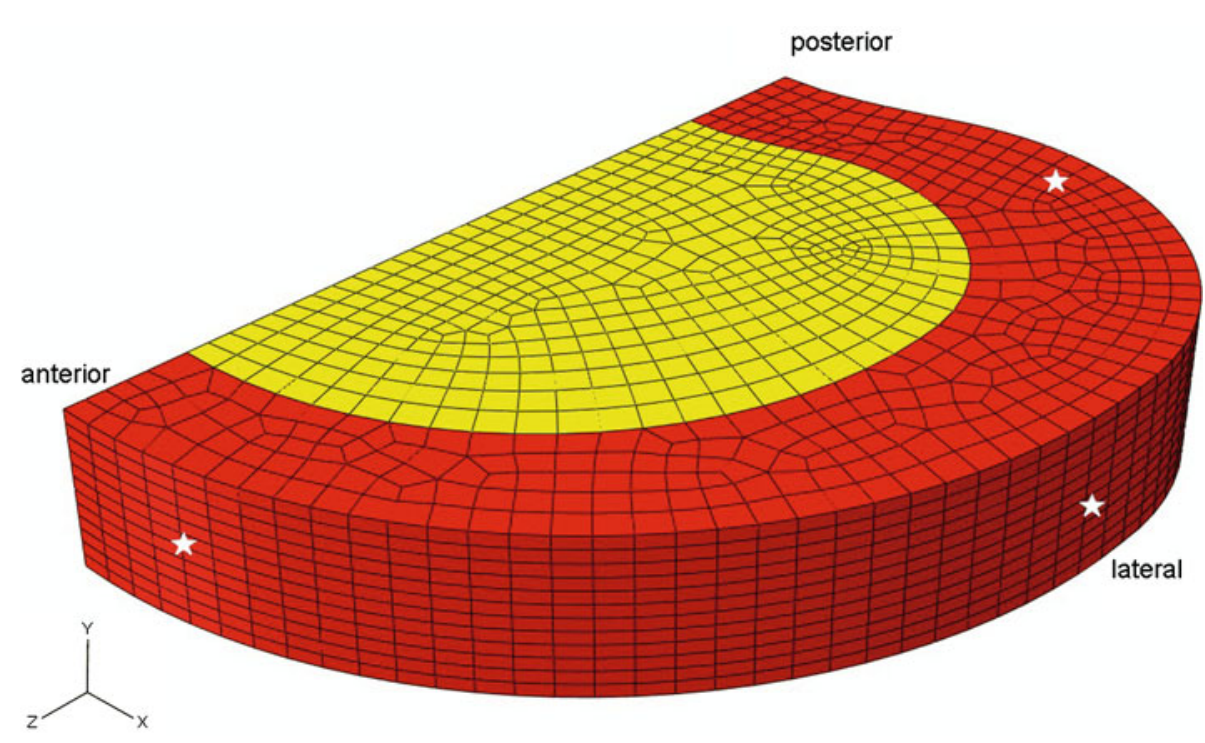

enhanced to account for smaller fibril structures. This adaptation is based on recent findings, which emphasize the importance of smaller fibril structures (Pezowicz et al. 2006a,b; Schollum et al. 2008; Veres et al. 2008) with regard to the annulus stiffness and structure.

The development of a whole-organ disc FE model is based on three pillars: anatomical geometry, constitutive laws and material laws. While the constitutive laws are well established, the material laws are based on numerical assumptions. Hence, verification with experimental data of several experiments is essential (Jones and Wilcox 2008). A recently published study (Heuer et al. 2007) on creep associated changes of human L4/5 discs provides such data (intradiscal pressure, height change and radial bulging) for verification purposes. The objective of this study was to evaluate if constitutive and material laws acquired on isolated samples of nucleus and annulus tissue could be used directly in a whole-organ 3D FE model to describe the behavior of the intervertebral disc.

\section{Material and methods}

\subsection{Finite element model}

The 3D FE mesh was based on a simplified geometry of a human lumbar disc (L4/L5; Schroeder et al. 2006). Because symmetry about the transversal and sagittal plane was assumed, the mesh was reduced to $1 / 4$ of the size of the disc (Fig. 1; Schroeder et al. 2006). The 3D OVED model describes the disc tissues as biphasic materials, consisting of porous solid matrix saturated with water. Additionally, the material properties are made directly dependent on the tissue composition. This is the same for all areas of the disc, i.e., the same for nucleus and annulus. Hence, the difference in biochemical composition of nucleus and annulus causes the material properties of both tissues to differ. The tissues are composed of the following constitutive components: elastic permeable non-fibrillar solid matrix, 3D viscoelastic collagen fiber structure and an osmotically pre-stressed extrafibrillar fluid (Schroeder et al. 2006, 2007; Schroeder et al. 2008). The total tissue stress is the sum of the three constituents.

The swelling behavior of the disc tissue is described through the biphasic swelling theory, as we assume the ion concentration to be in equilibrium at all times. The biochemical composition is accounted through the FCD in the swelling theory. The model includes a strain-dependent hydraulic permeability $(k)$, which depends on the amount of extrafibrillar water. It is implemented as follows (Maroudas and Bannon 1981; Schroeder et al. 2007; Wilson et al. 2006)

$k=\alpha\left(\frac{1}{1-n_{\mathrm{exf}}}\right)^{M}$

where $\alpha$ and $M$ are positive material constants, $k$ the permeability, and $n_{\text {exf }}$ the current extrafibrillar fluid fraction, which is dependent on strain.

The behavior of the non-fibrillar solid matrix is described through a modified Neo-Hookean law, while the viscoelastic behavior of the collagen fibers is represented through a Zener model. The constitutive law describing the non-fibrillar solid matrix is independent of the location in the disc, e.g., the same for nucleus and annulus. However, the fibril density and the solid volume fraction (i.e., accounting for the distinctive volumes of the components) are different at different locations in the disc. A more detailed description of the constitutive laws can be found elsewhere (Schroeder et al. 2006, 2007). 
The disc collagen fiber structure is a combination of larger collagen fibrils, arranged in concentric lamellae with alternating fiber orientation and smaller fibrils structures, e.g., minor collagen, elastin or collagen cross-links (Cassidy et al. 1989; Guerin and Elliott 2006b; Matcher et al. 2004; Schollmeier et al. 2000; Smith and Fazzalari 2006; Yu et al. 2005). In our previous studies, for simplicity, only two annular fiber orientations were assumed: $\pm 30^{\circ}$ to the transversal plane to represent the dominant fibril orientations in the lamellae (Schroeder et al. 2006). However, recent studies underline the importance of smaller fibril structures (Schollum et al. 2008; Veres et al. 2008) with regard to the annulus stiffness and structure. Hence, in the present analysis, the previous description of the 3D collagen network was supplemented with a second, more advanced approach. The description of the fiber structure within the model was kept as previously described (Wilson et al. 2005b). But now, secondary fibers were incorporated into the full 3D OVED model to account for smaller fibril structures. At each integration point, it was assumed that there were 2 primary fibers at an angle of \pm 30 degrees within the plane of the lamella, representing the dominant fiber orientation in annulus fibrosis tissue. Additionally, 13 secondary fibril directions are included to represent a random 3D network of fibrils, which is effectively represented by an isotropic fiber network at the level of our finite elements. Hence, these 13 fibers account for extra matrix stiffness in the $x$-, $y$ - and $z$-directions, and in all directions with angles of $45^{\circ}$ with respect to the $x-, y$-and $z$-axes. The density $\left(\rho_{c}\right)$ of the dominant primary fibrils is higher than that of the secondary fibrils, as represented by a $C$-value of 38 in the following formulas:

$$
\begin{aligned}
C & =\frac{d w_{1}}{d w_{2}} \Rightarrow d w_{1}=C d w_{2} \\
\rho_{c} & =\frac{d w_{1}}{d w_{\text {total coll }}}=\frac{C d w_{2}}{2 d w_{1}+13 d w_{2}}=\frac{C d w_{2}}{2 C d w_{2}+13 d w_{2}} \\
\rho_{c} & =\frac{C}{2 C+13} \text { for the primary fibrils } \\
\rho_{c} & =\frac{1}{2 C+13} \text { for the secondary fibrils }
\end{aligned}
$$

where $d w_{\text {total coll }}$ is the total dry weight of collagen, $d w_{1}$ is the dry weight of collagen in the primary fibril direction and $d w_{2}$ is the dry weight of collagen in the secondary fibril direction (Wilson et al. 2005b).

\subsection{Tissue properties-constitutive and biochemical composition}

The annulus was simulated with a collagen content of $60 \%$ per dry weight and with a fluid fraction of $77 \%$ per wet weight and a FCD of $0.15 \mathrm{mEq} / \mathrm{ml}$ (Sivan et al. 2006a; Skaggs et al. 1994). The nucleus had a collagen content of $9 \%$ per dry weight, a fluid fraction of $82.5 \%$ per wet weight and a
FCD of $0.3 \mathrm{mEq} / \mathrm{ml}$ extrafibrillar water (Sivan et al., unpublished data). As a result of the low collagen content and the assumption of no oriented collagen fiber structure in the nucleus pulposus, the tissue was described only through the elastic permeable non-fibrillar solid matrix and an osmotically pre-stressed extrafibrillar fluid. On the other hand, the annulus tissue with its distinctive 3D collagen structure was described as composed of all three constituents.

Tissue mechanical behavior tests on isolated samples were simulated with models incorporating the tissue composition to calculate the constituent parameter values (Schroeder et al. 2008). The non-fiber properties of the disc were determined by simultaneously fitting the model to the average experimental data from confined compression experiments of both nucleus and annulus tissue. Similarly, the fiber properties were defined using the uniaxial tensile tests in circumferential direction of the annulus at 6 and $10 \%$ strain (Johannessen and Elliott 2005; Schroeder et al. 2008). The curve-fitting procedures were performed iteratively, using a multidimensional unconstrained non-linear minimization procedure available in Matlab Version 5.3 (The MathWorks Inc.). From within this Matlab procedure, ABAQUS was called to simulate the different experiments. The output from ABAQUS was then transferred to Matlab, after which the objective function was determined. The iteration process was started with the following initial values for the non-fibrillar matrix; shear modulus $\left(G_{m}=1.23 \mathrm{MPa}\right)$, a positive material constant $(\alpha=$ $\left.1.964 \mathrm{e}^{-16} \mathrm{~m}^{4} / \mathrm{Ns}\right)$ and positive constant $M(M=1.57)$. Note, the hydraulic permeability was about a factor 3 higher than the fitted positive material constant $\alpha$, as the model accounts for the intra-extrafibrillar water differentiation. The initial values for the fibrillar matrix consisted of the parallel stiffness $\left(E_{0}=77.0 \mathrm{MPa}\right)$, the serial stiffness constant $\left(E_{\varepsilon}=\right.$ $500 \mathrm{MPa})$ and the damping coefficient $\left(\eta=1.8 \mathrm{e}^{3} \mathrm{MPa}-\mathrm{s}\right.$; Schroeder et al. 2008).

\subsection{Boundary conditions}

Simulation of the tissue mechanical behavior tests on isolated samples were performed with exactly the same boundary conditions, loading protocols and meshes as previously described in Schroeder et al. (2008). For simulations on the whole-organ level, similar boundary conditions were applied, as previously described in Schroeder et al. (2006). In brief, because of symmetry, we assumed no axial displacement and no fluid flux across the transversal and sagittal symmetry planes. Along the vertebra-disc interface, we assumed the anterior-posterior displacement and the lateral displacement to be negligible because the bone is much stiffer than the disc. For the same reason, we tied the axial displacement of all the nodes along the vertebra-disc interface to each other, allowing the distance between the two vertebrae to change. The exception was the disc-vertebra interface where outflow 
was simulated in a more realistic way, i.e., only free fluid flow at the nuclear tissue boundary but no fluid flow from the annular region. Initially, in each simulation, the model was allowed to equilibrate with a simulated isotonic salt solution $(0.15 \mathrm{M})$ such that the disc developed its normal pressure and fluid content (isotonic step).

Then, a recently published study (Heuer et al. 2007) on creep-associated changes of lumbar discs was simulated. Their experiments were performed on seven non to mildly degenerated human lumbar segments (L4-L5). In the experimental loading protocol, an axial compression load of $500 \mathrm{~N}$ was applied and maintained over $15 \mathrm{~min}$. The first measurements of intradiscal pressure, height change and radial bulging were taken at $60 \mathrm{~s}$. Several more measurements at different time points $(2,5,10$, and $15 \mathrm{~min})$ were also taken to describe the creep behavior over time. This was done similarly for the model simulations. To evaluate the creep response predicted by the model, the experimental protocol was simulated with different boundary conditions for the fluid flow. The flow boundaries were varied as follows; free out-inflow, no fluid out or inflow allowed and a resistance hampering the outflow on the vertebra-disc interface. The latter simulates a phenomenon often seen with in vitro experiments, the clogging of endplates. The clogging of endplates was simulated through creating a resistance by reducing the permeability $\left(k=1.0 \mathrm{e}^{-48} \mathrm{~m}^{4} / \mathrm{Ns}\right)$, in an extra thin layer of elements added on the vertebra-disc interface (Ayotte et al. 2001; MacLean et al. 2007; van der Veen et al. 2007).

\section{Results}

\subsection{Constitutive properties based on experiments of isolated tissue samples}

For the confined compression behavior of the nucleus and annulus, the reaction forces were generally in good agreement with the experimental range of the stress relaxation response (Figs. 2 and 3). However, the computed reaction force was overestimated during the ramp-up portion of the annulus tissue experiment (Fig. 3). Because these discrepancies did not allow a full fit for the annulus, we included only the computed reaction force after the strain ramp-up into the minimization procedure. Although the simulated initial relaxation rate was slightly faster than the experimental observation, the equilibrium values agreed well, and the entire response was within the experimental range. The calculated constitutive parameter values were shear stiffness $\left(G_{m}=1.65 \mathrm{MPa}\right)$, hydraulic permeability $\left(3 \times \alpha=1.767 \mathrm{e}^{-16} \mathrm{~m}^{4} / \mathrm{Ns}\right)$ and positive constant $(M=1.525)$ to describe the compressive behavior of normal human intervertebral disc tissues. The coefficients of correlation $\left(R^{2}\right)$ of the simulations with

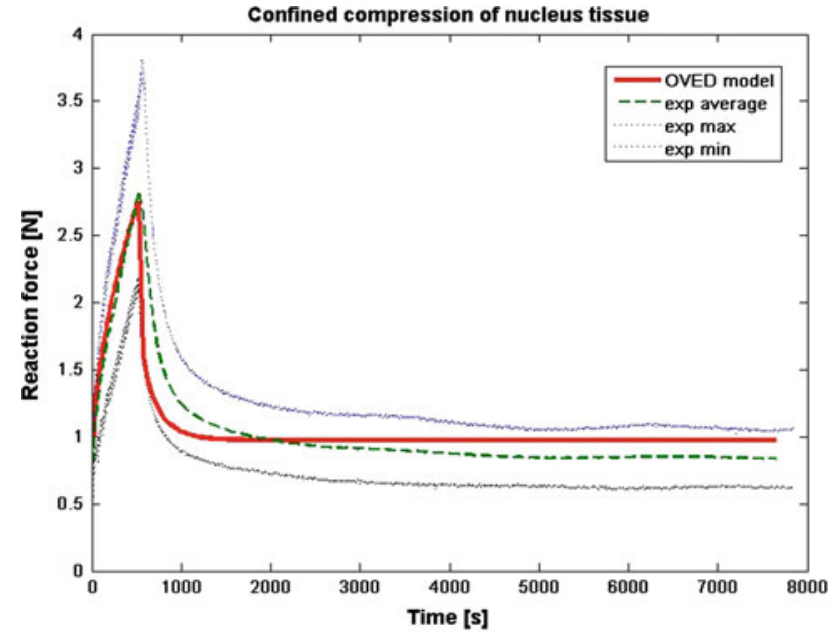

Fig. 2 Comparison of experimental reaction forces from confined compression experiment (Schroeder et al. 2008) and constitutive FE model simulations of nucleus tissue samples

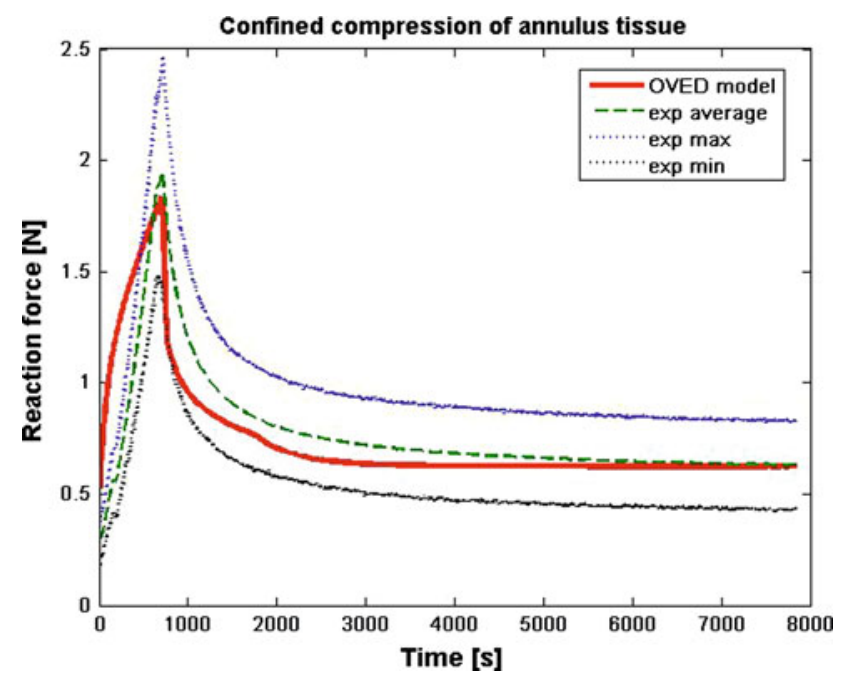

Fig. 3 Comparison of experimental reaction forces from confined compression experiment (Schroeder et al. 2008) and constitutive FE model simulations of annulus tissue samples

these values to the experimental data were 0.94 for the nucleus and 0.97 for the annulus.

For the two tensile tests with $6 \%$ strain and $10 \%$ maximum strain, the final simulations agreed generally well with the experimental data (Figs. 4 and 5). At both strain levels, the peak reaction force was overestimated with the error being larger for $10 \%$ strain. The simulated relaxation rates for $6 \%$ and $10 \%$ strain were respectively slower and faster than that of the experimental observation. Nevertheless, the final computed reaction forces for both tensile tests lay within the experimental range with coefficients of determination $\left(R^{2}\right)$ of 0.94 and 0.87 , respectively. As a result, the constitutive parameters parallel stiffness $\left(E_{0}=235 \mathrm{MPa}\right)$, the serial stiffness constant $\left(E_{\varepsilon}=4,141 \mathrm{MPa}\right)$ and the damping coefficient 


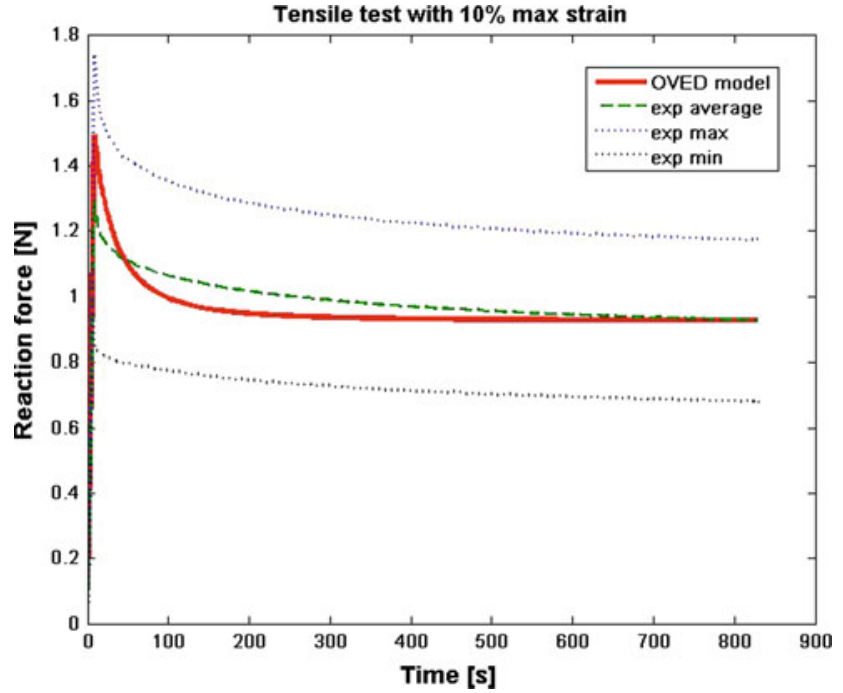

Fig. 4 Comparison of experimental reaction forces from uniaxial tensile test with $10 \%$ strain (Schroeder et al. 2008) along with constitutive FE model fit for annulus tissue samples

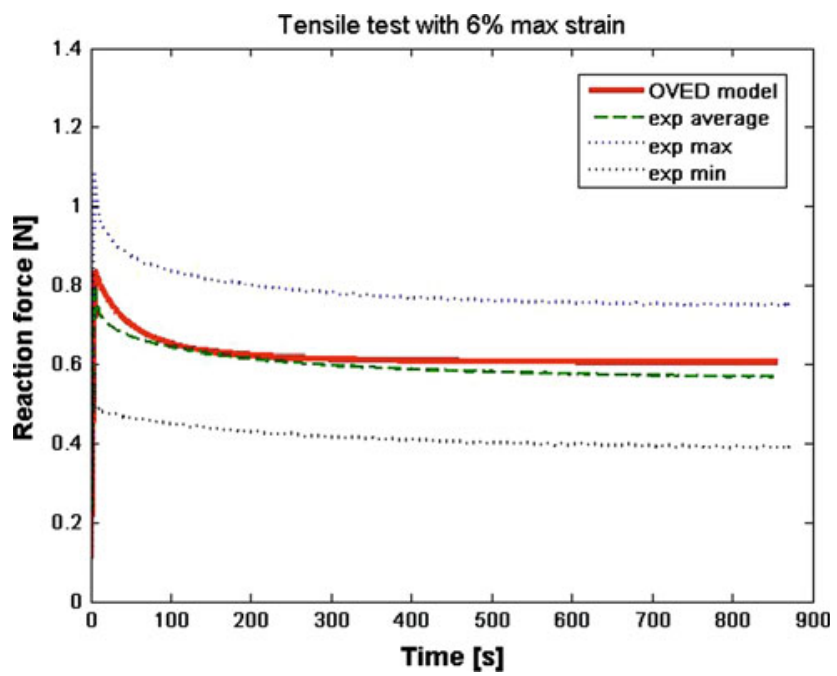

Fig. 5 Comparison of experimental reaction forces from uniaxial tensile test with 6\% strain (Schroeder et al. 2008) along with constitutive FE model fit for annulus tissue samples

$\left(\eta=10.3 \mathrm{e}^{3} \mathrm{MPa}-\mathrm{s}\right)$ were obtained to describe the viscoelastic behavior of the annulus collagen fibers.

\subsection{D disc behavior on whole-organ level}

For evaluation of geometrical changes at different time points, nodes in the anterior, posterolateral and lateral annulus region were selected (Fig. 1). At time point $60 \mathrm{~s}$, the axial load resulted in a computed height decrease of $1.11 \mathrm{~mm}$, an anterior radial bulging of $0.7 \mathrm{~mm}$, posterolateral bulge of $0.6 \mathrm{~mm}$ and lateral bulge of $0.66 \mathrm{~mm}$. At time point $900 \mathrm{~s}$, the model predicted an average radial deformation of $0.1 \mathrm{~mm}$ and a height reduction of about $0.746 \mathrm{~mm}$ (Table 1). An axial load of $500 \mathrm{~N}$ resulted in a total height reduction of $1.85 \mathrm{~mm}$ with normal flow boundary conditions. However, a change in the flow boundary conditions, e.g., first allowing no fluid outflow during the 15 min loading period resulted in a total height reduction of about $1.02 \mathrm{~mm}$, while a hampered fluid outflow through a resistance on the vertebra-disc interface resulted in a total height decrease of about $1.02 \mathrm{~mm}$ (Table 1).

The axial load of $500 \mathrm{~N}$ at $60 \mathrm{~s}$ resulted in a computed intradiscal pressure range of $0.2-0.6 \mathrm{MPa}$ (Fig. 6) for the nucleus. A linear decrease of the intradiscal pressure was noticed in the simulation during the 15-min loading period, resulting in an intradiscal pressure of $0.39 \mathrm{MPa}$ for the nucleus region at time point $900 \mathrm{~s}$ (Fig. 7). The change in the flow boundary conditions for both simulations (no outflow, resistance) resulted in an increased intradiscal pressure $0.4-0.8 \mathrm{MPa}$ (Fig. 7) for the nucleus region.

\section{Discussion}

The OVED model simulated confined compression and uniaxial tensile tests on isolated tissue samples, as well as the whole-organ disc behavior as measured by Heuer et al. (2007). This is possible, provided that secondary fiber structures, representing the small fibrils connecting the fibers within the lamellae and anchoring the lamellae with themselves, are accounted for. We were able to match the experimental results accurately by including a network of secondary fibers. With only the primary fiber network and isotropic properties for the non-fibrillar network, we were unable to obtain a suitable solution. The existence of such random fiber structure is supported by the morphological findings on the organization of collagen fibers in the annulus fibrosus (Elliott and Setton 2000; Pezowicz et al. 2006a,b; Schollum et al. 2008; Veres et al. 2008). However, it is well possible that with another approach, i.e., without assuming a secondary fiber network, similar results could have been obtained.

The fair agreement between model and experiment is obtained using constitutive material properties that are the same for annulus and nucleus. Annulus and nucleus tissue models differ in the 3D organ OVED model only by their composition and their fiber architecture.

The compressive properties of the poroelastic solid combined with the viscoelastic fiber properties show a good reproduction of all 4 experiments (confined compression experiments of nucleus and annulus tissue and uniaxial tensile tests at 10 and $6 \%$ strain of annulus tissue). Through a sensitivity analysis, the overestimation of the reaction force in the loading step of the annulus confined compression experiment as seen in Schroeder et al. (2008) was analyzed (Fig. 3). The possibility that the upper sample layer was damaged and leaching of PG occurred during sample preparation 
Table 1 Comparing creep response of OVED model with experimental results (Heuer et al. 2007) at different time points over $900 \mathrm{~s}$

\begin{tabular}{|c|c|c|c|c|c|}
\hline \multirow[t]{2}{*}{ Deformation (mm) } & \multirow[t]{2}{*}{ Time (s) } & \multicolumn{3}{|l|}{ OVED model } & \multirow{2}{*}{$\begin{array}{l}\text { Heuer et al. (2007) } \\
\text { In vitro BC }\end{array}$} \\
\hline & & Free fluid flow BC & No fluid flow BC & Resistance BC & \\
\hline Height change & 60 & -1.11 & -0.96 & -0.86 & $-(1.00-1.35)$ \\
\hline Anterior bulging & 60 & 0.70 & 0.81 & 0.69 & $0.46-1.34$ \\
\hline Posterolateral bulging & 60 & 0.60 & 0.62 & 0.53 & $0.24-0.90$ \\
\hline Lateral bulging & 60 & 0.66 & 0.70 & 0.60 & $0.10-1.09$ \\
\hline Ave. radial bulging & 900 & 0.1 & 0.06 & 0.01 & 0.1 \\
\hline Incremental height change & 900 & -0.74 & -0.06 & -0.16 & $-(0.08-0.26)$ \\
\hline Total height change & 900 & -1.85 & -1.02 & -1.02 & $-(1.08-1.57)$ \\
\hline
\end{tabular}

The experimental protocol was simulated with different boundary conditions for the fluid flow: free flow, no fluid flow allowed and a resistance hampering the flow on the vertebra-disc interface

Fig. 6 Color plot of an axially loaded model with $500 \mathrm{~N}$ at $60 \mathrm{~s}$ showing the intradiscal pressure distribution in nucleus and annulus. Low pressure indicates outflow boundaries for fluid

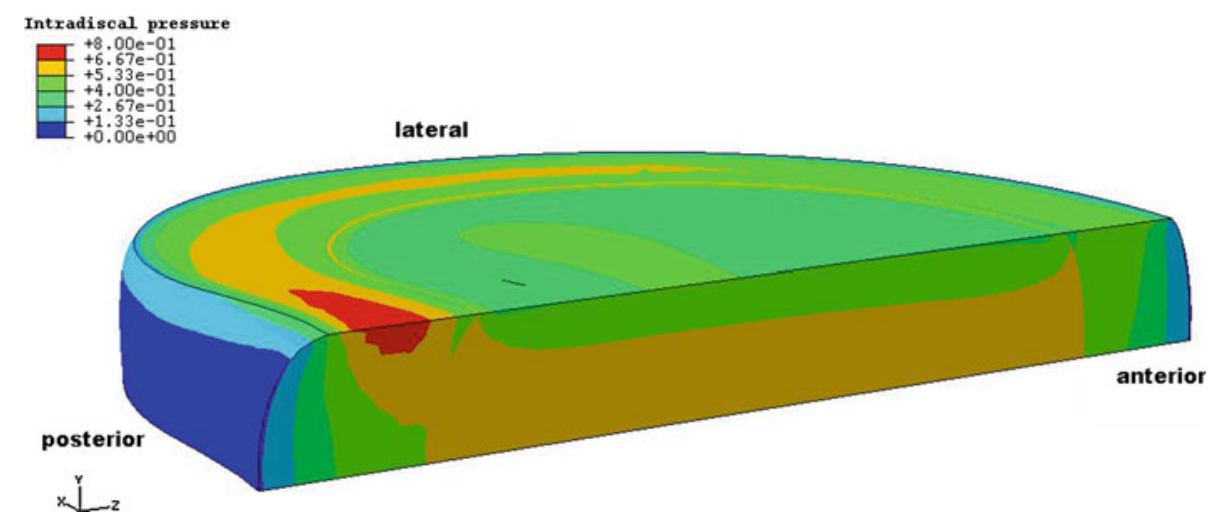

was explored by varying the biochemical composition in the first layer of the mesh adjacent to the porous platen. A reduction of the FCD by $50 \%$ suppressed the overestimation by half, supporting this hypothesis.

After the obtained material and constitutive laws of nucleus and annulus tissue were incorporated into the wholeorgan 3D OVED model, its overall behavior (intradiscal pressure, radial deformation and height change) agreed fairly well with in vitro creep experiments of human L4/L5 discs (Heuer et al. 2007). The computed intradiscal pressure range $(0.2-0.6 \mathrm{MPa})$ was in good agreement with the average intradiscal measurements $(0.36-0.53 \mathrm{MPa})$ at the first time point (60 s). Heuer et al. (2007) noticed a linear reduction of the intradiscal pressure (0.36-0.52 MPa) during the 900s creep period; a similar trend was predicted by the 3D OVED model (0.38-0.42 MPa; Fig. 7).

The predicted radial deformations and height change at time point $60 \mathrm{~s}$, lay well within the experimental range (Table 1). The same holds for the computed radial deformation (bulging) at time point $900 \mathrm{~s}$, which agreed well with the experimental data. During the creep phase (60-900 s), the predicted incremental height change $(-0.74 \mathrm{~mm})$ was overestimated by a factor 4 in comparison with the experimental measurements (0.08-0.26) of Heuer et al. (2007). Nevertheless, the total height reduction $(1.85 \mathrm{~mm})$ pre- dicted by the OVED model was in a reasonable range of the experimental measurements $(1.08-1.57 \mathrm{~mm})$. This differentiation, incremental height change measured at $60 \mathrm{~s}$ and the time-dependent creep behavior (60-900 s) was explicitly stated by Heuer et al. (2007), which allowed for this detailed verification of the time-dependent disc behavior.

The discrepancies between the model and experimental results might be related to the biphasic swelling theory, which is used to describe the swelling behavior of the tissue. The theory assumes that ion flux is infinitely fast, hence negligible. It has been shown by Wilson et al. (2005a) that the inaccuracy introduced by this simplification is relatively small for cartilaginous tissues, including disc. However, local readjustments of the ionic equilibrium may take some time, so that the model predictions in the first minutes of the loading may be inaccurate. Also, using a discrete number of fibers and assuming the primary fibril direction is constant with an angle of $\pm 30^{\circ}$ to the transversal plane is an approximation of the actual, slightly varying fiber angle in the annulus fibrosis (Holzapfel et al. 2005). This is of relevance in the tissue mechanical behavior test as the fiber orientation of the isolated samples was not measured directly but is based on other experimental studies (Guerin and Elliott 2006a; Skaggs et al. 1994).The simplified geometry of the 3D disc model, which 

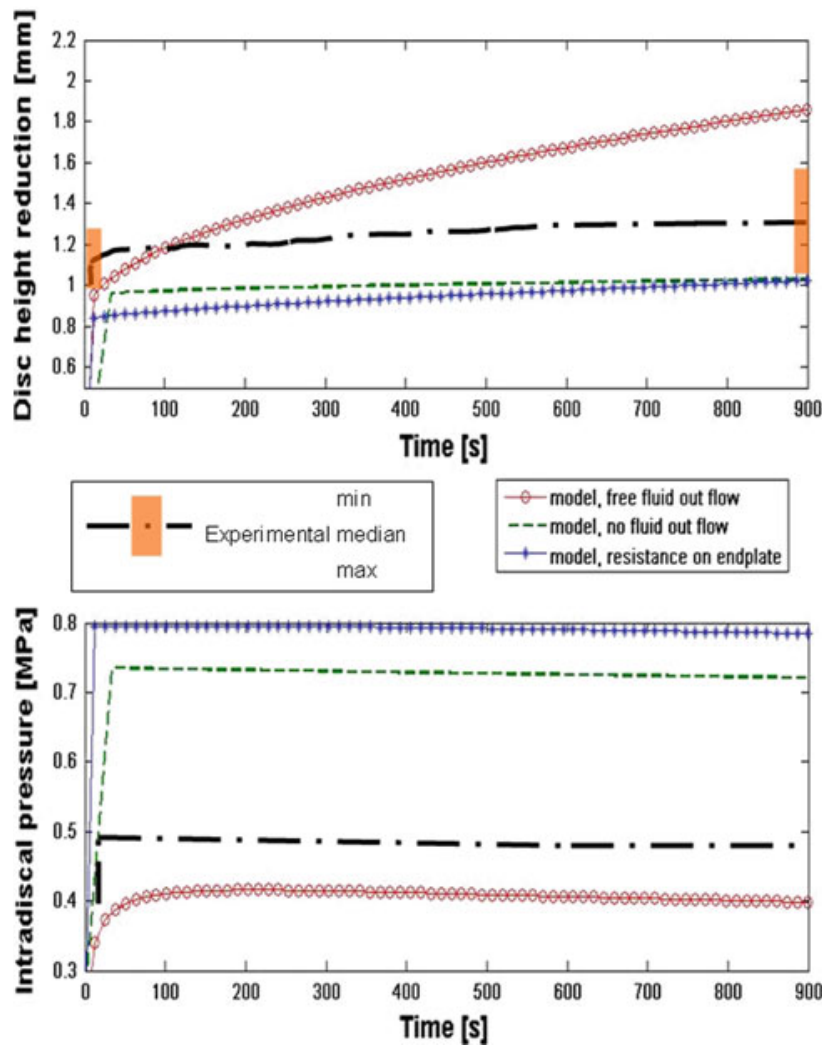

Fig. 7 Predictions for intradiscal pressure and disc height reduction of OVED model under same experimental conditions as by Heuer et al. (2007) (axial loading of $500 \mathrm{~N}$ over $900 \mathrm{~s}$ ). Simulations allowing fluid flow, no flow and resistance show clearly that most of the creep response predicted by the model is due to fluid outflow

does not account for the convex curvature of the endplates and their deformability (Brinckmann et al. 1983; Iatridis et al. 2005; MacLean et al. 2007) might have also influenced model predictions.

Properties (biochemical) were determined on samples from other studies which were then applied to discs of Heuer et al. (2007). Similarly, the small sample size used in the tissue tests could be a reasonable cause. Nevertheless, evaluation of the compressive disc properties calls for cautious interpretation of the results, as in vitro experiments may not be able to maintain the correct poroelastic properties or boundary conditions as experienced in vivo (Jones and Wilcox 2008). Several studies pointed out that the fluid flow through the endplate might be hampered through blood clotting in vitro (Adams and Hutton 1983; Ayotte et al. 2001; Broberg 1993; MacLean et al. 2007; van der Veen et al. 2005), creating a resistance. Variation of the outflow boundaries (free fluid flow, no fluid flow, flow resistance) on the vertebra-disc interface of the 3D OVED model allowed a closer evaluation of the predicted creep response. A simulation where no fluid flow is allowed (Fig. 7) showed that the predicted creep response of the model is largely influenced by the fluid outflow. Furthermore, the predicted creep response of the model $(1.02 \mathrm{~mm})$ compares well with the experimental range $(1.08-1.57 \mathrm{~mm})$, when the fluid flow is hampered through a resistance (clotting of blood in the endplates) on the vertebral-disc interface. In this line of reasoning, the predicted deformations and poroelastic disc properties of the $3 \mathrm{D}$ OVED model show a promising trend to correctly account for the complex biochemical and biophysical interaction of the native disc tissue, but without experimental assessment of this boundary condition, more detailed computational analysis is not warranted.

To conclude, the OVED model simulates isolated tissue experiments on confined compression and uniaxial tensile test, as well as the whole-organ disc behavior as measured by Heuer et al. (2007) provided that the simplified fiber structure from earlier studies was extended with a more complex fiber structure approach (smaller fibril structures). The composition-based modeling as presented in this paper presents the advantage of reducing the numbers of material parameters to a minimum and to use the measurable tissue composition directly as in input. Hence, this approach provides the possibility to describe internal mechanical conditions of the disc, which cannot be directly measured, as a function of assessable biophysical characteristics. This is relevant for current research of new treatment strategies, e.g. nucleus prosthesis, annulus repair or biological disc repair.

Acknowledgments This research forms part of the Project P2.01 IDiDAS of the research program of the BioMedical Materials institute, co-funded by the Dutch Ministry of Economic Affairs. This work was also funded by the "EURODISC" project of the European Union (grant no. QLK6-CT-2002-02582).

Open Access This article is distributed under the terms of the Creative Commons Attribution Noncommercial License which permits any noncommercial use, distribution, and reproduction in any medium, provided the original author(s) and source are credited.

\section{References}

Adams MA, Hutton WC (1983) The effect of posture on the fluid content of lumbar intervertebral discs. Spine 8:665-671

Adams MA, McNally DS, Dolan P (1996) 'Stress' distributions inside intervertebral discs, the effects of age and degeneration. J Bone Joint Surg Br 78:965-972

Argoubi M, Shirazi-Adl A (1996) Poroelastic creep response analysis of a lumbar motion segment in compression. J Biomech 29: 1331-1339

Ayotte DC, Ito K, Tepic S (2001) Direction-dependent resistance to flow in the endplate of the intervertebral disc: an ex vivo study. J Orthop Res 19:1073-1077

Brinckmann P, Frobin W, Hierholzer E, Horst M (1983) Deformation of the vertebral end-plate under axial loading of the spine. Spine $8: 851-856$

Broberg KB (1993) Slow deformation of intervertebral disks. J Biomech 26:501-512

Cassidy JJ, Hiltner A, Baer E (1989) Hierarchical structure of the intervertebral disc. Connect Tissue Res 23:75-88 
Chen J, Baer AE, Paik PY, Yan W, Setton LA (2002) Matrix protein gene expression in intervertebral disc cells subjected to altered osmolarity. Biochem Biophys Res Commun 293:932-938

Cheung JT, Zhang M, Chow DH (2003) Biomechanical responses of the intervertebral joints to static and vibrational loading: a finite element study. Clin Biomech (Bristol Avon) 18:790-799

Elliott DM, Setton LA (2000) A linear material model for fiber-induced anisotropy of the anulus fibrosus. J Biomech Eng 122:173-179

Guerin HA, Elliott DM (2006a) Degeneration affects the fiber reorientation of human annulus fibrosus under tensile load. J Biomech 39:1410-1418

Guerin HL, Elliott DM (2006b) Quantifying the contributions of structure to annulus fibrosus mechanical function using a nonlinear, anisotropic, hyperelastic model. J Orthop Res 25:508-516

Heuer F, Schmitt H, Schmidt H, Claes L, Wilke HJ (2007) Creep associated changes in intervertebral disc bulging obtained with a laser scanning device. Clin Biomech (Bristol Avon) 22: 737-744

Holzapfel GA, Schulze-Bauer CA, Feigl G, Regitnig P (2005) Single lamellar mechanics of the human lumbar anulus fibrosus. Biomech Model Mechanobiol 3:125-140

Houben GB, Drost MR, Huyghe JM, Janssen JD, Huson A (1997) Nonhomogeneous permeability of canine anulus fibrosus. Spine 22: $7-16$

Iatridis JC, Laible JP, Krag MH (2003) Influence of fixed charge density magnitude and distribution on the intervertebral disc: applications of a poroelastic and chemical electric (PEACE) model. J Biomech Eng 125:12-24

Iatridis JC, MacLean JJ, Owen JP (2005) Role of endplates in contributing to compression behaviors of motion segments and intervertebral discs. In: ECM VI/SRN I: spinal motion segment: from basic science to clinical application, Davos, $\mathrm{CH}$

Johannessen W, Elliott DM (2005) Effects of degeneration on the biphasic material properties of human nucleus pulposus in confined compression. Spine 30:E724-E729

Jones AC, Wilcox RK (2008) Finite element analysis of the spine: towards a framework of verification, validation and sensitivity analysis. Med Eng Phys 30:1287-1304

MacLean JJ, Owen JP, Iatridis JC (2007) Role of endplates in contributing to compression behaviors of motion segments and intervertebral discs. J Biomech 40:55-63

Marchand F, Ahmed AM (1990) Investigation of the laminate structure of lumbar disc anulus fibrosus. Spine 15:402-410

Maroudas A, Bannon C (1981) Measurement of swelling pressure in cartilage and comparison with the osmotic pressure of constituent proteoglycans. Biorheology 18:619-632

Matcher SJ, Winlove CP, Gangnus SV (2004) The collagen structure of bovine intervertebral disc studied using polarization-sensitive optical coherence tomography 131. Phys Med Biol 49:1295-1306

Natarajan RN, Andersson GB (1999) The influence of lumbar disc height and cross-sectional area on the mechanical response of the disc to physiologic loading. Spine 24:1873-1881

Neidlinger-Wilke C, Wurtz K, Urban JP, Borm W, Arand M, Ignatius A, Wilke HJ, Claes LE (2006) Regulation of gene expression in intervertebral disc cells by low and high hydrostatic pressure. Eur Spine J 15(Suppl 15):372-378

Op De Beeck R, Hermans D (2000) Research on work-related low back disorders. European agency for safety and health at work

Pezowicz CA, Robertson PA, Broom ND (2006a) The structural basis of interlamellar cohesion in the intervertebral disc wall. J Anat 208:317-330

Pezowicz CA, Schechtman H, Robertson PA, Broom ND (2006b) Mechanisms of anular failure resulting from excessive intradiscal pressure-a microstructural-micromechanical investigation. Spine 31:2891-2903
Rohlmann A, Zander T, Schmidt H, Wilke HJ, Bergmann G (2006) Analysis of the influence of disc degeneration on the mechanical behaviour of a lumbar motion segment using the finite element method. J Biomech 39:2484-2490

Roughley PJ (2004) Biology of intervertebral disc aging and degeneration: involvement of the extracellular matrix. Spine 29: 2691-2699

Ruberte LM, Natarajan RN, Andersson GBJ (2009) Influence of single-level lumbar degenerative disc disease on the behavior of the adjacent segments-A finite element model study. J Biomech 42:341-348

Schmidt H, Kettler A, Rohlmann A, Claes L, Wilke HJ (2007) The risk of disc prolapses with complex loading in different degrees of disc degeneration-A finite element analysis. Clinical Biomechanics 22:988-998

Schollmeier G, Lahr-Eigen R, Lewandrowski KU (2000) Observations on fiber-forming collagens in the anulus fibrosus. Spine 25: 2736-2741

Schollum ML, Robertson PA, Broom ND (2008) ISSLS Prize Winner: Microstructure and Mechanical Disruption of the Lumbar Disc Annulus Part I: A Microscopic Investigation of the Translamellar Bridging Network. Spine 33:2702-2710

Schroeder Y, Wilson W, Huyghe JM, Baaijens FP (2006) Osmoviscoelastic finite element model of the intervertebral disc. Eur Spine J 15(Suppl 15):361-371

Schroeder Y, Sivan S, Wilson W, Huyghe JM, Maroudas A, Baaijens FPT (2007) Are disc pressure, stress and osmolarity affected by intra and extrafibrillar fluid exchange? J Orthop Res 10:1317-1324

Schroeder Y, Elliott DM, Wilson W, Baaijens FP, Huyghe JM (2008) Experimental and model determination of human intervertebral disc osmoviscoelasticty. J Orthop Res 26:11411146

Sivan S, Merkher Y, Wachtel E, Ehrlich S, Maroudas A (2006a) Correlation of swelling pressure and intrafibrillar water in young and aged human intervertebral discs. J Orthop Res 24:12921298

Sivan S, Neidlinger-Wilke C, Wurtz K, Maroudas A, Urban JP (2006b) Diurnal fluid expression and activity of intervertebral disc cells. Biorheology 43:283-291

Skaggs DL, Weidenbaum M, Iatridis JC, Ratcliffe A, Mow VC (1994) Regional variation in tensile properties and biochemical composition of the human lumbar anulus fibrosus. Spine 19:1310 1319

Smith LJ, Fazzalari NL (2006) Regional variations in the density and arrangement of elastic fibres in the anulus fibrosus of the human lumbar disc. J Anat 209:359-367

Urban JP, Maroudas A (1981) Swelling of the intervertebral disc in vitro. Connect Tissue Res 9:1-10

Urban JP, McMullin JF (1985) Swelling pressure of the inervertebral disc: influence of proteoglycan and collagen contents. Biorheology 22:145-157

Urban JP, Roberts S (2003) Degeneration of the intervertebral disc. Arthritis Res Ther 5:120-130

Urban JP, Maroudas A, Bayliss MT, Dillon J (1979) Swelling pressures of proteoglycans at the concentrations found in cartilaginous tissues. Biorheology 16:447-464

van der Veen AJ, Mullender M, Smit TH, Kingma I, van Dieen JH (2005) Flow-related mechanics of the intervertebral disc: the validity of an in vitro model. Spine 30:E534-E539

van der Veen AJ, van Dieen JH, Nadort A, Stam B, Smit TH (2007) Intervertebral disc recovery after dynamic or static loading in vitro: is there a role for the endplate. J Biomech 40: 2230-2235

Veres SP, Robertson PA, Broom ND (2008) ISSLS prize winner: microstructure and mechanical disruption of the Lumbar disc annulus 
part II: how the annulus fails under hydrostatic pressure. Spine 33:2711-2720

Wilson W, van Donkelaar CC, Huyghe JM (2005a) A comparison between mechano-electrochemical and biphasic swelling theories for soft hydrated tissues. J Biomech Eng 127:158-165

Wilson W, van Donkelaar CC, van Rietbergen B, Huiskes R (2005b) A fibril-reinforced poroviscoelastic swelling model for articular cartilage. J Biomech 38:1195-1204

Wilson W, Huyghe JM, van Donkelaar CC (2006) A compositionbased cartilage model for the assessment of compositional changes during cartilage damage and adaptation. Osteoarthritis Cartilage $14: 554-560$
Wognum S, Huyghe JM, Baaijens FP (2006) Influence of osmotic pressure changes on the opening of existing cracks in 2 intervertebral disc models. Spine 31:1783-1788

Wu JS, Chen JH (1996) Clarification of the mechanical behaviour of spinal motion segments through a three-dimensional poroelastic mixed finite element model. Med Eng Phys 18:215-224

Yu J, Fairbank JC, Roberts S, Urban JP (2005) The elastic fiber network of the anulus fibrosus of the normal and scoliotic human intervertebral disc. Spine 30:1815-1820 